\title{
CUTANEOUS TUBERCULOSIS AS A TRIGGER AND MIMICFOR A SEVERE BEHÇET'S DISEASE - CASE REPORT
}

Sean Hideo Shirata Lanças", , , Matheus Zanata Brufatto', Laura Maria Silva de Siqueira', Douglas Squizatto Leite', Luiz Eduardo Valente', Fabio Vicente Leite', Henrique Pereira Sampaio', Andrea de Almeida Peduti Batista', Sula Glaucia Lage Drumond Pacheco'

1.Universidade Estadual Paulista, Botucatu (SP), Brazil.

*Corresponding author: sslancas@hotmail.com

\section{BACKGROUND}

Various autoimmune inflammatory diseases share clinical syndromes similar to each other, and can be mimicked by secondary causes such as infections, drugs or neoplasms, what makes the diagnosis challenging and prospective. We presented a case accompanied in our service with an initial diagnosis of extrapulmonary tuberculosis; however, with a follow-up diagnosis of a severe Behçet's disease.

\section{CASE REPORT}

L.E.R., a 24-year-old male, admitted in September 2017 for investigation of a clinical presentation of an 8-month diarrhea without pathological products, recurrent night fever, weight loss, asymmetric peripheral oligoarthritis and erythematous-necrotic nodular cutaneous lesions in lower limbs, in addition to reports of sporadic painful oral and genital ulcers. The initial investigation focused on infections, inflammatory bowel diseases, Behçet's disease and spondyloarthritis. Colonoscopy showed serpiginous shallow ileocolonic ulcers and aphthoid rectal ulcers, both with histologically nongranulomatous eosinophilic infiltrate. He had moderately high inflammatory markers, nonerosive arthritis, and negative HLAB-27 and pathergy test. Without improvement with antibiotics and corticotherapy, a subsequent diagnosis of cutaneous tuberculosis was made by evaluation of the skin lesions biopsy, and treatment with RIPE scheme was initiated, alongside tapering corticoids regarding a possible associated Poncet's syndrome. Diarrhea and fever ceased, he regained weight and evolved with cutaneous lesions healing. However, despite successful tuberculosis treatment, there were recurrent flares of arthritis, colitis, acute anterior uveitis, dactylitis and acneiform skin lesions during corticosteroids tapering, all fully responsive to prednisone and methotrexate. In addition to that, he evolved with prominent anxiety and behavioral changes. In February 2020, during further corticosteroids tapering, there was recurrence of the nodular cutaneous lesions, fever, arthritis and diarrhea, along with worsening anxiety and visual hallucinations. With a new biopsy without evidences of tuberculosis and showing inflammatory infiltrate suggestive of perivasculitis pattern, a definitive Behçet's disease diagnosis was made. We proceed with methylprednisolone pulse therapy, high dose of tapering corticosteroids, associated with infliximab infusions, with a fast improvement of all symptoms, behavior and functionality, with no recurrences since then.

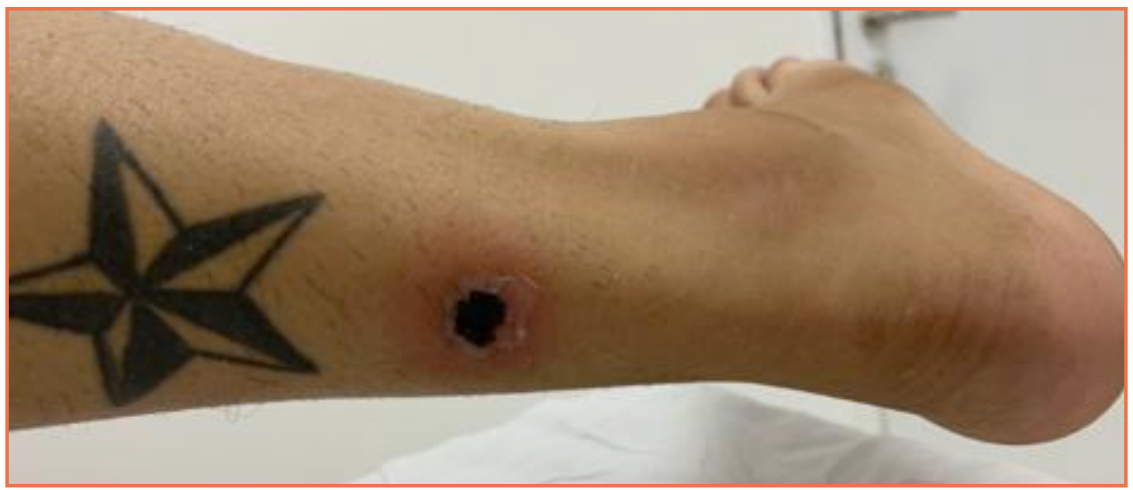

Figure 1. Erythematous necrotic nodular lesion during patient recurrence. Pathological anatomy showing a neutrophilic inflammatory infiltrate suggestive of perivasculitis.

\section{CONCLUSION}

The case emphasizes the importance of the follow-up diagnosis in front of the overlap between the clinical manifestations of the rheumatological syndromes, beyond interposition with secondary causes as possible mimickers or triggers. 